\title{
The Thermodynamics of Supercritical Fluids
}

$S^{w}$

JPERCRItical fluids are gases at pressure and temperatures slightly above those of the vapour-liquid critical point. At this point, liquid and vapour become indistinguishable, and the vapour pressure curve of the liquid ends. The main distinctive characteristic of the supercritical fluid condition is that the density of the fluid is very sensitive to small changes in pressure and temperature. Actually, at the critical point, the compressibility is infinite.

Density is directly related to many other physical (and chemical) properties of a fluid. The most important in supercritical fluid applications is the solvent power, that is, the ability to dissolve other substances.
At gas-like densities, a supercritical fluid can hardly dissolve anything. When mixed with a liquid or a solid, the partial pressure of the condensed substance in the gas phase is limited to its vapour pressure, because the density of the supercritical fluid is too small to allow the interactions that lead to dissolution. On the contrary, at liquid-like densities, the molecules of the fluid can cluster around the molecules of the other substance and bring them into the gas phase.

So, with small variations of pressure or temperature, a supercritical fluid can be brought to a density where it dissolves (reasonably) high amounts of a solute or to another one where it precipitates (almost) everything it had dissolved before.
The following schematic diagram depicts these events. (compression / decompression could be replaced by cooling/heating).

This cycle is the basis of many of the hitherto devised applications of supercritical fluids (although by no means does it summarise them all).

An important aspect of the cycle is that the solvent power of the SCF varies continuously with density. Therefore, on the left side of the cycle, if a high density (high pressure) is used, high amounts of different solutes may be dissolved. On the contrary, if a moderate density (moderate pressure or high temperature) is used, the solvent power will be lower, but the selectivity can be much higher,

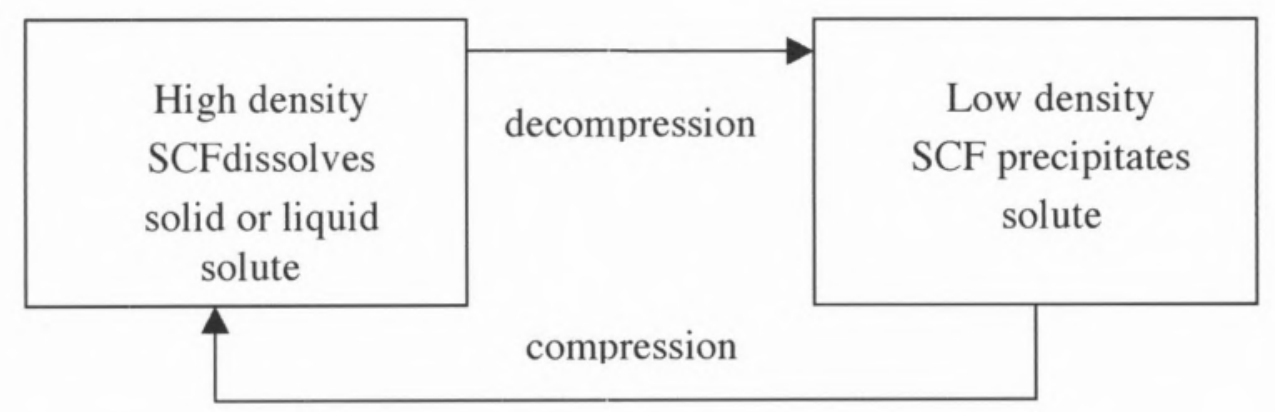

*Faculdade de Ciências e Tecnologia, Universidade Nova de Lisboa 2825-516 Caparica, Portugal mnponte@itqb.unl.pt 
that is, only a few solutes, ideally one, will dissolve. On the right side, precipitation can be modulated, if, for instance, the decompression will proceed in steps. In principle, the less soluble solutes will precipitate in the first step, and more soluble ones in further successive decompression steps.

Many SCF extraction and fractionation processes have been devised in the recent past, which successfully use these principles.

Critical pressures of known substances are (much) higher than atmospheric pressure, and so a supercritical fluid is always a gas at high pressures. Fluid phases at high pressures are a popular subject in Jorge Calado's scientific family. He supervised several doctoral theses on this subject, including mine. His main inspiration in this area was William B. Streett, Professor at the West Point Academy and later at the School of Chemical Engineering, Cornell University, Ithaca, New York. The photograph in Figure 1 shows the entrance of Olin Hall, where the School is located.

Quite obviously, the thermodynamics of the place are very different from Lisbon's, where we have not had any snow for 50 years. But a real and fruitful collaboration was established and lasted for many years. Long and short stays in Ithaca became a habit for Jorge's collaborators (and especially for Jorge Calado himself, of course), where they became familiar with all aspects of highpressure technology. OAlthough supercritical fluids were not explicitly included in Streett and Calado's interests, the combination of expertise in high-pressure thermodynamics with multiple prospective applications became too strong an attraction for several of their collaborators. Since the mid-eighties, research on supercritical fluids started in earnest, and it could be argued that it is currently one of the most successful of the research activities of the Family (Jorge Calado's scientific family, as given in the family tree shown in this issue). More than a quarter of all doctoral theses have now addressed this subject, and the current number of graduate students (candidates to become Family members) prepa- ring theses on supercritical fluids.is almost half of the total.

The appeal of the field for a thermodynamicist is the variety of the phase behaviour in mixtures where one of the components is close to the critical point, especially the number and composition of phases in equilibrium in these conditions. Moreover, this behaviour is very sensitive to changes in pressure and temperature. Understanding the effects of these variables is therefore a core issue in all supercritical fluid processes.

When two liquids are mixed, the vapour pressure of the mixture at a given temperature varies with the composition. In Figure 2, a typical phase diagram for a mixture of two liquids is shown on the left side. The temperature is lower than the critical temperature of any of the pure liquids, so both of them have a vapour pressure. Each of these is represented on its axis of ordinates of the diagram. Between these two limits lie all possible mixtures - composition in mole fraction $x$ for component 1 and $(1-x)$ for component 2. Raoult's law predicts a vapour pressure of the mixtures given by a straight line between the vapour pressures of the two pure liquids. The diagram also gives information on the composition of the vapour phase produced by evaporation of a given liquid mixture. The two compositions -evaporating liquid and resulting vapour - are connected by a horizontal tie-line, as shown in the diagram for two different pressures.

If the temperature is above the critical temperature of one of the components, we have a mixture of a liquid with a supercritical gas, and the phase diagram is of the type shown on the right side. As component 1 is above its critical temperature, it has no vapour pressure, and the phase separation area cannot intercept the $x=1$ ordinate. The gas-rich phase compositions reach a maximum value in $\mathrm{x}$, which corresponds to a minimum value of the solubility of the liquid in the supercritical gas, and then turn back. The liquid-rich phase, on the other hand, rapidly incorporates more and more gas, up to a maximum ${ }^{1}$ pressure where the two phases reach equal

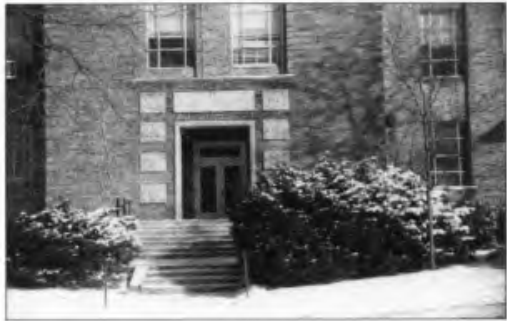

figure 1 Entrance to Olin Hall, School of Chemical Engineering, Cornell University

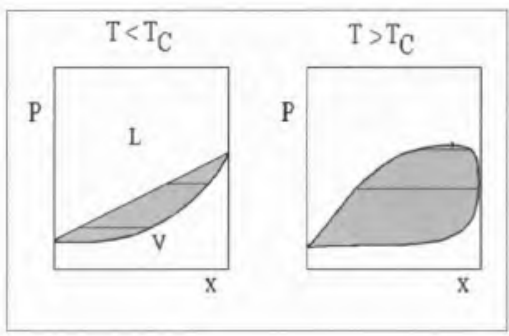

figure 2 Schematic fluid phase equilibrium diagrams for mixtures of: left side - two liquids; right side - a liquid and a supercritical fluid.

composition - the critical pressure of the mixture at the given temperature.

An important feature shown by the diagram is that the effect of pressure is drastic on the liquid phase, provoking a very large increase of the amount of gas dissolved. In fact, close to the critical pressure, the liquid is composed of 80 $\%$ molar or more of the supercritical component. The properties of this type of liquid are profoundly changed in relation to those of the initial liquid pure component. A whole new area of supercritical fluid applications is based on these modified properties and on the advantages they confer, for example, to carry out chemical reactions or to crystallise and encapsulate solutes by rapid expansion.

On the contrary, the solubility of the liquid in the supercritical gas remains small up to high pressures, and only increases significantly very close to the critical pressure.

Another important feature apparent in the diagram is that, above the critical pressure, at the given temperature, the mixture presents only one fluid phase, 


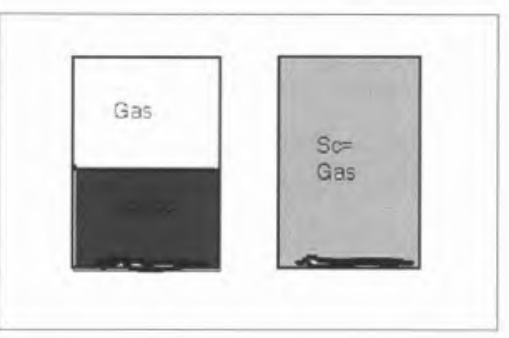

figure 3 Biphasic system Us. one phase supercritical system in contact with solid catalyst

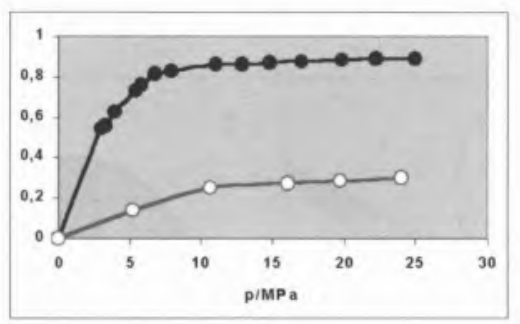

figure 4 Viscosity reduction of liquid Poly(ethylene glycol) ( $M W=400)$, represented by closed circles and expressed as a fraction of the viscosity of the pure polymer, as a function of pressure of carbon dioxide. The open circles represent the composition of the liquid, in mole fraction of carbon dioxide.

and the components freely mix in all proportions. The critical pressure therefore separates a region where the mixture can present phase separation from another one where it can only appear in a single fluid phase. Depending on the desired application, the design of a process may require the mixture in one state or the other-separation processes require two phases, chemical reaction may proceed faster in a single phase, for instance.

The knowledge of how many phases does a mixture present for a given set of parameters (pressure, temperature, composition) is therefore vital in supercritical fluid technology.

Imagine, a case where a chemical reaction is carried out by contact between reactants in two immiscible phases, for instance the hydrogenation of a liquid, where hydrogen is always very scarcely soluble in the liquid phase. These reactions are often catalysed by a metal. Mass transfer of hydrogen through the liquid phase, towards the solid metal, controls the reaction rate. This is a difficult process, with an additional disadvantage - low concentrations of hydrogen in the vicinity of the catalyst may promote undesirable side reactions.

If a supercritical fuid is used as solvent of the whole reaction mixture, there are cases where only one gas phase contacts the catalyst, as shown in Figure 3.

Many authors have followed the strategy of looking for suitable conditions (pressure, temperature, composition) to carry out reactions in the conditions depicted on the right side of Figure 3. As pointed out above, the usually low solubility of most substances in supercritical fluids makes these endeavours quite difficult, that is, there are very few systems that fulfil those conditions and are interesting from the point of view of industrial application.

Surprisingly, work from our laboratory [1] has shown that hydrogenation of alpha-pinene in the presence of a palladium catalyst, in high-pressure carbon dioxide, proceeds faster in three-phase (liquid + gas + solid catalyst) conditions than in a two-phase (supercritical mixture + catalyst) system. This is in contradiction with the accepted wisdom that a supercritical, one-phase mixture allows a faster diffusion of hydrogen towards the catalyst, yielding faster rates of reaction. The explanation is that supercritical $\mathrm{CO}_{2}$ is highly soluble in the liquid pinene, as it is in most terpenes. At pressures close to the critical pressure, the liquid phase in a biphasic mixture may contain $80 \mathrm{~mol} \%$ or more of $\mathrm{CO}_{2}$. This "expanded" liquid can dissolve much higher amounts of a gas reactant, like hydrogen, that would be otherwise possible. Access of hydrogen to the catalyst is no longer the controlling rate step.

Another illustration of this modification of the properties of a liquid by contact with a supercritical gas was the collaboration study, carried out by our group and João Fareleira's group at IST, about the properties of Poly-(ethyleneglycol)s $+\mathrm{CO} 2$ systems [2,3].

At low to moderate temperatures, the phase equilibrium behaviour in these systems is similar to the one pictured in
Figure 2. However, when the pressure goes over a certain value, not much higher than the critical pressure of carbon dioxide, the gas solubility in the liquid reaches a limiting value. The separation of phases remains, at least up to very high pressures.

The viscosity of the pure polymer is high. The measurements performed at IST of the viscosity when carbon dioxide is added to the polymer indicate a drastic reduction, which can go up to almost $90 \%$ of the initial viscosity (that is, the viscosity of polymer + high pressure $\mathrm{CO}_{2}$ is about $10 \%$ of the viscosity of the pure polymer). This viscosity reduction is closely parallel to the amount of carbon dioxide dissolved in the liquid polymer, as depicted in Figure 4.

Once again, phase equilibrium controls the properties. The implications for facilitating the processing of highly viscous materials are quite obvious.

Exploiting the possibilities offered by these "expanded" liquids, resulting from the dissolution of supercritical carbon dioxide in liquids or melts, is a promising line of research. High-pressure phase equilibrium measurements are the key element. The methods developed by Lionel Staveley, at ICL, Oxford, Bill Streett, at West Point and Cornell, NY, and Jorge Calado, at IST, are still our guideline in this whole new area.

\section{References}

[1] D. Chouchi, D. GOurgouillon, M. Courel, J. Vital, M. Nunes da Ponte "The influence of phase behaviour on reactions at supercritical conditions: the hydrogenation of a-pinene* Ind. Eng. Chem. Research, 2001, 40, 2551

[2] D. Gourgouillon, H.M.N.T. Avelino, J.M.N.A. Fareleira, M. Nunes da Ponte "Simultaneous Viscosity and Density Measurement of Supercritical CO2 - saturated PEG 400* J. Supercritical Fluids 1998, 13, 177

[3] D. Gourgouillon, M. Nunes da Ponte "High Pressure Phase Equilibria for Poly(ethyleneglycol)s + CO2: Experimental Results and Modelling" Phys. Chem. Chem. Phys. 1999, 1, 5369 